\title{
Soccer at the Microscale: Small Robots with Big Impact
}

\author{
S. L. Firebaugh ${ }^{1}$, J. A. Piepmeier ${ }^{1}$ and C. D. McGray ${ }^{2}$ \\ 1 United States Naval Academy \\ ${ }^{2}$ Semiconductor Electronics Division, National Institute of Standards and Technology \\ USA
}

\section{Introduction}

The robots in the RoboCup Nanogram demonstration events are the size of dust, and compete on a millimeter-scale playing field under a microscope (Fig. 1). It seems unlikely that the robots of the Nanogram events will meet the RoboCup goal of beating the human World Cup champion team, so what is the point? The potential for these robots in applications beyond soccer is vast, but so are the engineering challenges. The Nanogram challenge provides an intermediate target for microroboticists, and the innovation that has resulted has been astounding. Some day heart surgery may be as simple as swallowing a pill of microrobots only a few generations evolved from a microscale soccer team.

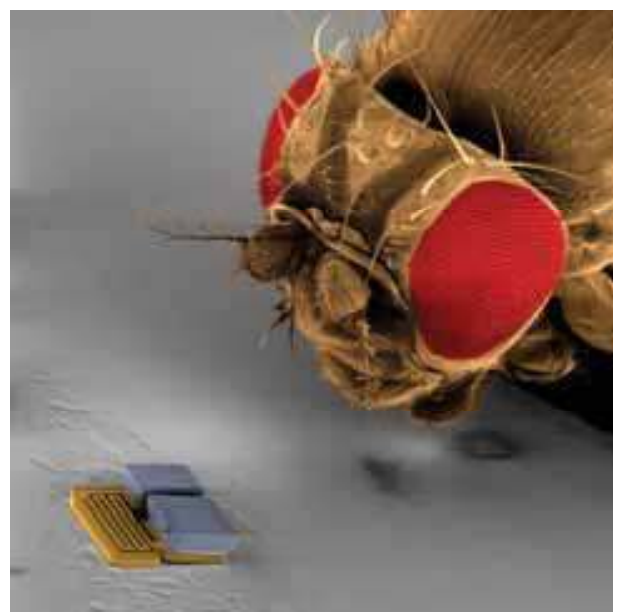

Fig. 1. The Magmite robot, developed by ETH Zurich, in relation to a fruit fly, as captured in a scanning electron micrograph (Vollmers et al., 2008; Kratochvil et al., 2009; Frutiger et al., 2008; Frutiger et al., 2009). The Magmite has participated in the RoboCup Nanogram events since their inception. Figure used with permission from the authors. 
The field of microrobotics has been defined "to include the robotic manipulation of objects with characteristic dimensions in the millimeter to micrometer range (micromanipulation) as well as the design and fabrication of robotic agents in a smilar size range (microrobots)" (Abbott et al., 2007). This distinguishes the robots being discussed in this chapter from robots on the millimeter to centimeter scale that are sometimes called "microrobots," but would more accurately be termed "miniature robots" (Dario et al., 1992).

The fundamentals of traditional robotics - including kinematics, dynamics, motion planning, computer vision, and control (Spong et al., 2006) -- apply soundly to the field of microrobotics. However, the nature of the actuators that function best within the microdomain often dominates how these fundamental concepts are applied. Microrobots competing in the Nanogram events have utilized electrostatic and magnetic actuation methods. Thermal, vibratory, piezoelectric, and biological actuation have also been used for microrobotics, and so these technologies are also reviewed. While microscale end effectors or grippers have been made, Nanogram participants have only utilized passive designs to aid in ball handling. To date, sensors and controllers are all off-board for the Nanogram League, and the control strategies used with the various microrobots are discussed below.

\subsection{Microrobot Applications}

The classic example of robot applications is the set of line assembly tasks performed in the manufacture of large durable goods, such as automobiles, where robots have been used commercially for decades. Yet these days, robots are rapidly moving into other niche markets such as surgery, security, and entertainment at a pace that has raised the eyebrows of many industry leaders and technology visionaries. As robots become smaller, cheaper, more versatile, and more reliable, some analysts have forecast the coming of a robotics industry of large enough scope to mirror in the next thirty years the economic and societal impact of the computer revolution thirty years ago (Gates, 2007). In response, Microsoft has established a robotics product line, the EU is doubling its investment in robotics research, and U.S. lawmakers have formed a new congressional caucus on robotics.

Some of the most exciting future applications of robotics will require systems that are many orders of magnitude smaller than today's commercial robots. These micro-robots would have masses and characteristic dimensions that are best measured in nanograms and micrometers, respectively. Their tiny size would allow them to penetrate small pores and cavities and to explore microscopic environments, such as the vascular system of the human body. As with today's transistors, the material costs for such small robots are insignificant, so building a million need not be much more expensive than building a dozen. This opens up the possibility of disposable, massively parallel robotic systems for search and rescue in disaster sites, exploration of planetary surfaces or military encampments, and other as yet unimagined tasks.

\subsection{Challenges of the Microscale}

Realization of commercially viable microrobots to meet these application requirements will require solutions to numerous engineering challenges. At the heart of these challenges lies the fact that the physical forces that dominate locomotion and manipulation tasks in micro- 
and nano-scale size regimes are different from those most relevant at larger scales. For objects much below a millimeter in their characteristic dimensions, surface phenomena such as friction, adhesion, heat transfer, and electrostatic attraction become significantly more important, while inertia, weight, heat capacity, and other body phenomena become comparatively less important (Madou, 1997). Furthermore, these surface phenomena can change whenever two micro-scale parts come into contact as a result of triboelectric charging and other factors. These phenomena can be difficult to measure, but must be tracked and controlled to prevent failure modes such as altered transfer functions and irreversible adhesion.

With surface forces dominating motion at these scales, adsorption of contaminants - even water - can lead to devastating results. Proper environmental control can be critical, especially for electrostatic devices (Liu, 2006). Devices and operating environments must be kept meticulously clean, and humidity should be regulated. In contrast to larger robots, once a microrobot is damaged it can rarely be fixed and must instead be discarded. These devices must therefore be manufactured in quantity, at comparatively low cost. While the very small size of these devices makes low-marginal-cost manufacturing practical, it also makes it very difficult to identify damaged devices prior to critical failure. The ability to identify a damaged device in time to replace it before it fails can be a crucial difference between a winning team and a losing one.

Even the very smallest of batteries available today remain far too big to be mounted on a microrobot. As a result, providing a source of power to these devices is a significant problem. One promising approach is to provide a source of wireless power that welldesigned microrobots can receive from their environment. This wireless power can be electrostatic (Donald et al., 2003), optical (Sul et al., 2006), magnetic (Vollmers et al., 2008), vibrational (Yasuda et al., 1994), or alternative modes yet to be developed. When multiple (competing) teams are involved, this ambient power must be provided in a way that will not unintentionally (or intentionally!) interfere with the operation of opposing teams. On-board power systems remain a challenging possibility, with thin-film batteries becoming increasingly energetic (Patil et al., 2008) and nuclear power presenting a feasible and exciting approach (Lal et al., 2005).

Control strategies familiar to robot coordination must be carefully reconsidered for applicability to the new field of microrobotics. For example, the closed-loop feedback approach to systems engineering is widely considered a necessity in micro-scale and smaller robots, but the optical systems often employed for this feedback at the macro scale can be inappropriate to micro-environments (Abbot et al., 2007). The depth and breadth of field under the microscope can be limiting factors for vision systems; it is often not possible to employ stereo cameras; and the pin-hole camera models widely used at macro-scales break down under the microscope (Abbot et al., 2007). Other feedback mechanisms must be similarly adapted. For example, in macro-scale actuators, servoing systems are often implemented with optical feedback from diodes or lasers, but capacitive feedback may be more effective at the micro- and nano-scale (Yamahata et al., 2008). 
Communication poses particular difficulties in micro-soccer competitions, where individual robots may be smaller than the wavelength of the radio signals typically used to communicate with their larger brethren. If an ambient power source is used, communication signals can be embedded in the power waveform, to be mechanically decoded by the microrobots' physical structure (Donald et al., 2006; Donald et al., 2008). New communication methods must be devised, and standards must be established to divide the available bandwidth between competing teams of multiple microrobots.

\subsection{RoboCup Nanogram Events}

Microrobotic soccer competitions have been used to drive innovation in microelectromechanical systems (MEMS) and microrobotic technologies and to provide educational opportunities. Games were held annually from 2007 through 2009 in association with the RoboCup Federation. Teams competed in each of three events:

- $\quad$ The Two-Millimeter Dash

- The Slalom Drill

- $\quad$ The Ball Handling Drill

In the two-millimeter dash, microrobots must demonstrate maximum speed and the ability to start and stop on command. The microrobots are placed onto a field of play that contains two open goals that are 500 micrometers deep, with two millimeters of space between opposite goal lines. A microrobot is placed inside one of the goals so that the entire body of the microrobot is behind the goal line. Upon a signal from the event timing system, the microrobot must sprint to the opposite goal line, stop inside the goal, and signal completion to the timing system.

The slalom drill proceeds in the same fashion as the two-millimeter dash, except that the area between the goal lines is obstructed by obstacles formed from thick-film photoresist. The microrobots must be maneuverable enough to navigate a path between the obstacles into the opposite goal.

To compete in the ball handling drill, microrobots must be capable of controlled planar pushing manipulation of small disks whose diameter is only 100 micrometers. The microrobot begins inside one of the goals as in the case of the other two events, and the space between the goals is obstructed as in the slalom drill. Tiny "micro-soccer balls," which consist of a $100 \mu \mathrm{m}$ diameter disk of silicon, are placed amongst the obstacles. Upon a signal from the event timing system, the microrobot moves out onto the playing field to push the micro-soccer balls into the opposing goal.

\section{Electrostatic Microrobots}

In the field of micro-electromechanical systems (MEMS), electrostatic transducers have long been a favored class of actuator (Kovacs, 1998). Forces are generated between insulated bodies having opposite net charge, resulting in motion. Electrostatic actuators can be comparatively easy to build using established microfabrication techniques, and they can generate considerable forces on micro-scale components due to large surface area to volume ratios. Classic electrostatic actuators from the field of MEMS include comb drive actuators 
(Tang et al., 1990), rotary side-drive micro-motors (Fan et al., 1988), and scratch drive actuators (Akiyama et al., 1993). Several teams have used robots that rely on electrostatic actuation in RoboCup Nanogram events, including the United States Naval Academy, Simon Fraser University, and Carnegie Mellon University, whose robots from the 2007 competition in Atlanta are shown in Fig. 2 below.
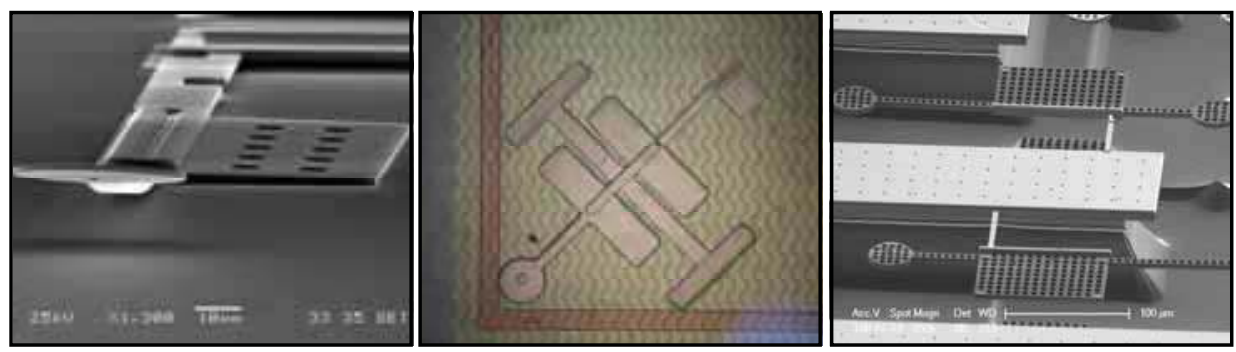

Fig. 2. Electrostatic actuators from the 2007 RoboCup Nanogram event. Left: The microrobot from the U.S. Naval Academy team (Firebaugh and Piepmeier, 2009), based on work by Donald et al. (Donald et al., 2006). Center: A polymer micromachined electrostatic microrobot from Simon Fraser University. Photo courtesy of Dan Sameoto. Right: CMOSMEMS electrostatic microrobots from Carnegie Mellon University. Photo courtesy of Fernando Alfaro, Chiung Lo and Professor Gary Fedder.

\subsection{Electrostatic Actuation}

The forces generated by electrostatic actuators can be predicted using simple capacitive circuit models. The energy stored in a capacitor of capacitance $C$ at voltage $V$ is given by:

$$
U_{c}=\frac{1}{2} C V^{2}
$$

Therefore, the force generated in a given direction, $r$, is therefore given by:

$$
F=\left(\frac{V^{2}}{2}\right) \frac{\partial C}{\partial r}
$$

Knowing the geometry of an electrostatic actuator allows us to approximate its capacitance, either analytically or using finite element methods. For example, in the simplest case of a parallel plate capacitor, the capacitance is $\varepsilon w L / g$, where $L$ is the length of the overlapping area of the two plates, $w$ is the width of the overlap, $g$ is the gap between the plates, and $\varepsilon$ is the permittivity of the material in the gap. If the plates are misaligned so that $L$ would be increased by shifting the top plate along the $x$ axis, then the $x$-component of the electrostatic force on the top plate would be $F_{x}=\varepsilon w V^{2} / 2 g$. Similarly, the force of attraction between the two plates is $F_{z}=\varepsilon w L V^{2} / 2 g^{2}$.

The force of attraction between the capacitor plates is a non-linear function of the plate separation. This leads to the useful phenomenon of snap-down voltages (Nathanson et al., 
1978). Imagine that the top plate were suspended over the bottom plate by a linear spring of constant $K$. For a given extension, $z$, the energy stored in the spring is:

$$
U_{S}=K \frac{z^{2}}{2}
$$

The energy stored in the capacitor, $U_{c}$, is:

$$
U_{C}=\frac{\varepsilon L w V^{2}}{2\left(g_{0}-z\right)}
$$

where $g_{0}$ is the plate separation at zero spring extension. Minimizing the energy of the system, the relationship between the plate separation and the applied voltage can be calculated as follows:

$$
V=\frac{g \sqrt{2 \varepsilon L w K\left(g_{0}-g\right)}}{\varepsilon L w}
$$

This function defines the curve shown in Fig. 3. To the right of the maximum voltage, the system is statically stable. To the left, the system is unstable and will collapse, extending the spring until the plates come into contact or encounter a hard stop. Solving for $V$ and maximizing with respect to $g$ produces the voltage and gap at which this snap-down event occurs (Nathanson et al., 1978):

$$
\begin{aligned}
V_{S D} & =\sqrt{\frac{8 K g_{0}^{3}}{27 \varepsilon L w}} \\
g_{S D} & =\frac{2 g_{0}}{3}
\end{aligned}
$$

If the plates are prevented from electrical contact by, for example, a layer of insulation, they will remain snapped down until the voltage is reduced below the release voltage. The release voltage for a given gap can be determined from the left-hand side of the graph in Fig. 3. Each hysteresis band between a snap-down voltage and a release voltage can be used as a mechanical memory bit for electrostatic microrobotic systems (Donald et al., 2006), allowing the robots to be configured as simple state machines without any on-board transistors. 


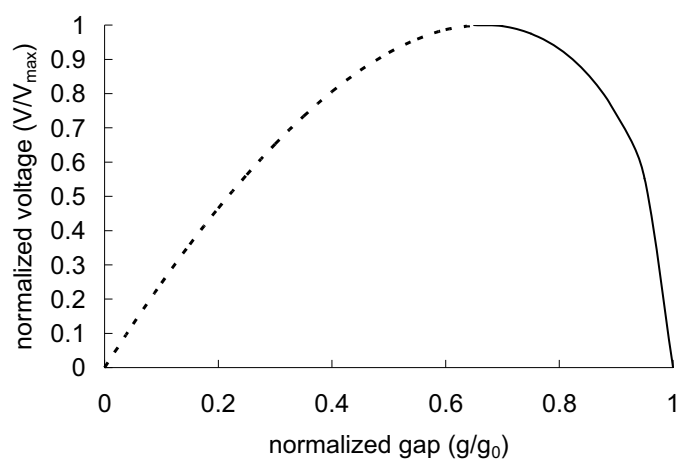

Fig. 3. Graph of capacitor plate separation versus applied voltage. To the right of the maximum, the system is statically stable. To the left, the system is unstable, and will collapse, extending the spring until the plates come into contact or encounter a hard stop. After Nathanson (Nathanson et al., 1978).

For microrobotic applications, one of the more common electrostatic actuators is the scratch drive. A scratch drive actuator (Akiyama et al., 1993) is composed of a thin polysilicon plate with a support at the front end. The plate is typically in the range of $60 \mu \mathrm{m}$ to $80 \mu \mathrm{m}$ on a side, and $1 \mu \mathrm{m}$ to $2 \mu \mathrm{m}$ thick. The support height is typically in the $1 \mu \mathrm{m}$ to $2 \mu \mathrm{m}$ range. The operation of the scratch drive is shown in Fig. 4 . When a voltage is applied between the polysilicon plate and the substrate beneath it, the plate is drawn down into flat contact with the dielectric layer. Since the front of the plate is supported by the bushing, strain energy is stored in the plate, and the edge of the bushing is pushed forward. When the voltage is removed, the strain is released, and the scratch drive plate snaps back to its original shape, slightly in front of where it began. When a periodic pulse is applied, this cycle is continuously repeated, and the scratch drive moves forward in a step-wise manner, achieving maximum speeds on the order of millimeters per second.

Steering can be accomplished by exploiting snap-down and release voltages of cantilever beams mounted on the scratch drive body. Fig. 2 (left) shows one such steering cantilever with a two-micron-diameter stylus at the tip. If the periodic drive pulse of the scratch drive actuator nests within the hysteresis band of these cantilevers, the cantilevers' position (raised or lowered) can be controlled independently from the operation of the actuator (Donald et al., 2006). 


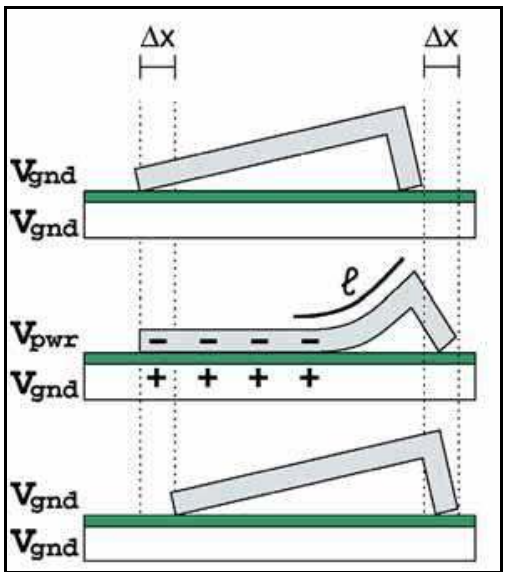

Fig. 4. A schematic of the operation of a scratch drive actuator (Akiyama et al., 1993). The length of the curved region of the plate, $\ell$, and the step size, $\Delta x$, are determined by the voltage.

\subsection{Electrostatic Microrobot Fabrication}

Fabrication of thin-film electrostatic actuators like the scratch drive is performed through photolithographic techniques that were originally developed for the integrated circuit industry. Each device is composed of multiple layers, each of which is defined by a lithographic mask. Typically, a material such as silicon, glass, or metal is deposited by chemical or physical vapor deposition and is then coated with a film of photoresist. The photoresist is exposed through a lithographic mask and developed to remove the unwanted portion of the film. The pattern defined by the remaining film is transferred into the material layer through an etching process, and then the residual photoresist is removed. As this process is repeated, the device is built up layer by layer. The equipment required for these processes is available at microfabrication laboratories in many universities. In addition, many standard thin-film microfabrication processes are available commercially as multiproject wafer services, where each wafer is divided between many researchers, producing significant cost decreases (Markus et al., 1995; Sniegowski et al., 1996; Tea et al., 1997). Participants in these processes receive a set of die containing devices built from their own supplied designs. The die can then be post-processed for additional customization if desired (Donald et al., 2006; Huikai et al., 2002).

\subsection{Electrostatic Microrobot Control}

The work by Donald (Donald et al., 2006) uses the pre-image motion planning strategy developed by Lozano-Perez, Mason, and Taylor (Lozano-Perez, et al., 1984). The planner starts with the goal position and computes backwards the sequence of single velocity motion primitives that leads to the initial position of each robot. This method assumes perfect robot motion. Since robots rarely live up to this assumption, a closed-loop error correction method is employed. The trajectory is recalculated at periodic intervals, making on-the-fly adjustments for observed error. In the case of (Donald et al., 2006) the feedback is 
provided by a digital microscope camera, and trajectory adjustments are made by changing the relative percentages of the interleaved control primitives.

The microrobotic devices may by modeled as nonholonomic mobile robots similar to a Dubins vehicle (Dubin, 1957) limited to forward motion and left turns. The state of the robot $q$ is given by:

$$
q=\left[\begin{array}{l}
x \\
y \\
\theta
\end{array}\right]
$$

where $(x, y)$ is the location of the robot, and $\theta$ is the orientation of the device. The kinematics of the device are given by:

$$
\dot{q}=\left[\begin{array}{c}
v \cos \theta \\
v \sin \theta \\
\sigma \omega \theta
\end{array}\right]
$$

where $v$ is a positive velocity with an expected constant value that is related to the stepping frequency in the forward motion wave form shown in Figure 7. The angular velocity $w$ is also a fixed value, and $\sigma \in\{0,1\}$ is the state of the steering arm $(0=$ up, $1=$ down). It should be noted that $w$ may be positive or negative depending on the construction of the arm. Without loss of generality, we will assume the value is positive for the remainder of this discussion. The robot is globally controllable, but not small time locally controllable (STLC).

As discussed in (G. Lionis and K.J. Kyriakopoulos, 2007) the radius of curvature can be varied by alternating the relative percentage of the two voltage waveforms that moves the robot either forward or in a turn within a short time period. The device kinematics of the ideal system can be decomposed using control vectors that characterize all of the possible motions of the scratch drive actuators. For scratch drive actuators, these vectors are given by the following

$$
g_{1}=\left[\begin{array}{c}
v_{1} \cos \theta \\
v_{1} \sin \theta \\
0
\end{array}\right], g_{2}=\left[\begin{array}{c}
v_{2} \cos \theta \\
v_{2} \sin \theta \\
\omega_{2}
\end{array}\right]
$$

A key departure here from the work (G. Lionis and K.J. Kyriakopoulos, 2007) is the absence of the ability to achieve a $-w$ or $-v$. The work in (G. Lionis and K.J. Kyriakopoulos, 2007) considered a larger microrobot with piezo-actuated legs similar to that discussed in (Martel, 2005). That system had the ability to go forwards and backwards as well as turn both left and right with fixed radius turns. Despite these differences, the large portions of the discussion of (G. Lionis and K.J. Kyriakopoulos, 2007) are still applicable. Of note is the analysis concerning a pulse width modulated (PWM) control strategy. The approach is to alternate between two of the permissible vectors. Fig. 5 shows the two waveforms that would be sent to the scratch drive actuator in order to switch between forward motion and turning motion. 


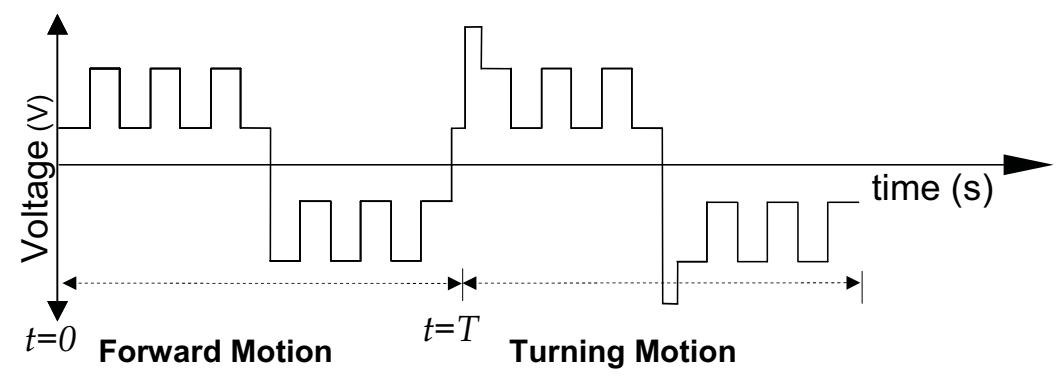

Fig. 5. Pulse width modulated (PWM) control scheme that alternates between forward motion for time $T$ and turning motion with time $a T$.

To create a PWM control scheme, a switching function is defined

$$
\sigma(t, a, T)=\left\{\begin{array}{cc}
1 & 0 \leq t<T \\
0 & T \leq t<(a+1) T \\
\sigma(t-(a+1) T, a, T) & t>(a+1) T
\end{array}\right.
$$

Physically, the switching is achieved by lowering and raising the steering arm. The control input is defined as $C_{g_{2}-g_{1}}^{a}\left(q_{0}\right)$, and is constructed by selecting $a, T, g_{1}$, and $g_{2}$ such that

$$
\dot{q}=\sigma(t, a, T) g_{1}+(1-\sigma(t, a, T)) g_{2}
$$

Note that $T$ defines the time for the motion primitive $g_{1}$ and $a$ controls the length of time for the second motion primitive $g_{j}$ relative to $g_{i}$.

It was shown in (G. Lionis and K.J. Kyriakopoulos, 2007) that as $T \rightarrow 0$, the microrobot with a fixed turning radius will move as a unicycle; a device capable of arbitrary curvature. For example, switching between $g_{1}$ and $g_{2}$ will result in

$$
g_{12 a}=\left[\begin{array}{c}
v \cos \theta \\
v \sin \theta \\
\frac{a}{a+1} \omega_{2}
\end{array}\right]
$$

While these results hold in the ideal case, implementation issues for scratch drive actuators violate two of the assumptions made in the analysis. First, the motion of the robots can be inconsistent. Devices often exhibit mild turning characteristics even when they are controlled by the straight motion wave function (Donald et al., 2006). Secondly, the 
practicalities of waveform generation dictate that $T$ is small, but does not necessarily approach zero. In (Donald et al., 2006) the length of $T$ was set at $0.25 \mathrm{~ms}$. In (Piepmeier, 2010) it was shown that the following model gives a better approximation of the motion of the device for larger control periods, $T$. The following vector field provides a closer approximation of the motion produced by a PWM-controlled scratch drive device.

$$
\tilde{g}_{12 a}=\left[\begin{array}{c}
\left(\frac{v_{1}}{a+1}+\frac{v_{2} a}{a+1}\right) \cos \theta \\
\left(\frac{v_{1}}{a+1}+\frac{v_{2} a}{a+1}\right) \sin \theta \\
\frac{1}{a+1} \omega_{1}+\frac{a}{a+1} \omega_{2}
\end{array}\right]
$$

\section{Magnetic Microrobots}

Two groups participating in the RoboCup Nanogram events, one from Carnegie Mellon University, and the other from ETH Zurich University, have demonstrated magnetic microrobots. These systems have shown less sensitivity to environmental variation than microrobots based on scratch drive actuators. The two magnetic microrobotic systems operate on different principles, as described below.

The Carnegie Mellon microrobot, developed by Steven Floyd, Chytra Pawashe, and Metin Sitti, is simply a laser machined slug of neodymium-iron-boron, a hard magnetic material, which is manipulated through externally applied magnetic fields (Floyd et al., 2008 Pawashe, 2008; Pawashe, 2009, a; Pawashe, 2009, b). This robot is similar to the microrobots developed by a number of groups for biomedical applications (Gauthier \& Piat, 2004; Yesin et al., 2006; Tamaz et al., 2008; Yamazaki et al., 2004). Robot technologies such as this, where the robot itself is a simple shape that is translated through externally applied magnetic fields without internally moving parts, will be termed "ferromagnetic-core-based robots." Most of these microrobot technologies are targeted towards operation in a three-dimensional fluid environment, such as the human body. The distinction for the Carnegie Mellon microrobot is that the control system has been tailored to allow the robot to operate on a surface.

The ETH Zurich microrobot (Vollmers et al., 2008; Kratochvil et al., 2009; Frutiger et al., 2008, a; Frutiger et al., 2008, b; Frutiger et al., 2009) utilizes an alternating magnetic field to excite mechanical resonances within the structure, allowing the robot to move through a stick-slip method. Bidirectional motion is facilitated by a $z$-axis clamping force. Robots of this type will be termed "resonant magnetic robots." Before describing these technologies in more detail, however, it is useful to review the physics of magnetics. 


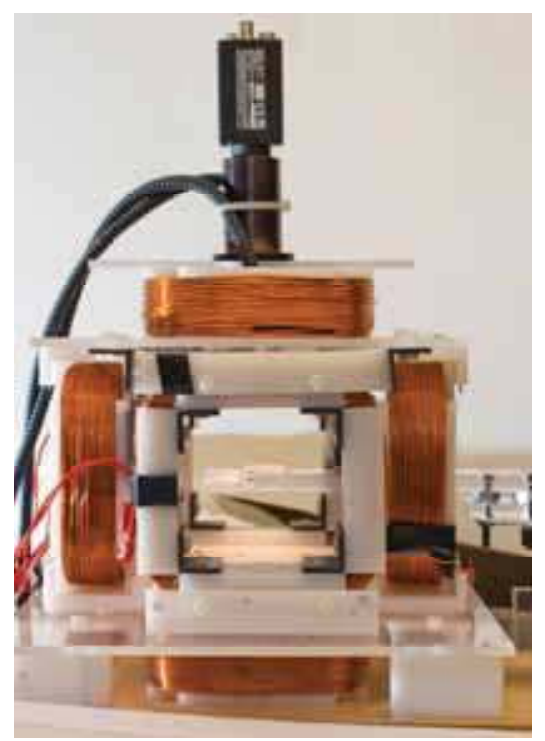

Fig. 6. Electromagnetic cage used by Floyd et al. for microrobot actuation (Floyd, 2008; Pawashe, 2008; Pawashe, 2009, a; Pawashe, 2009, b). Figure used with permission from the authors.

\subsection{Magnetic Force Generation}

For both of these technologies, the playing field area is encased inside of a cage of independent electromagnet coils, as is illustrated in Fig. 6 for the Carnegie Mellon microrobot. The Biot-Savart law determines the relationship between the current, $I$, through a coil, C, consisting of $\mathrm{N}$ turns, and the magnetic field density vector, $\mathrm{B}$, at a distant position (Hayt \& Buck, 2006):

$$
\vec{B}=\frac{\mu_{0} I}{4 \pi} \oint_{C} \frac{d \vec{L} \times \vec{a}_{R}}{R^{2}}
$$

where $\mu_{0}$ is the permeability of free space, $\mathrm{d} \mathbf{L}$ is an infinitesimal line segment along the direction of integration, $\mathbf{a}_{\mathbf{R}}$ is the unit vector pointing from $\mathrm{d} \mathbf{L}$ to the point of interest, and $R$ is the distance from the line segment to the point of interest. Of course, in a case where multiple independent coils are used, the net magnetic field at the microrobot location must be found by adding the contributions from each coil at the location of the microrobot. The important thing to note about this equation is that the field intensity is inversely proportional to the square of the distance between the coil and the robot. This is an important consideration for the control of the robot, as the resulting force is highly non-linear with position.

It's important to distinguish between magnetic field intensity vector, $\mathbf{H}$ (units: $A / m$ ), and the magnetic field density vector, $\mathbf{B}$ (units: $\mathrm{T}$ or $\mathrm{Wb} / \mathrm{m}^{2}$ ), which is the induced total magnetic field within a given material (Liu, 2006). The relationship between the two quantities is given by: 


$$
\vec{B}=\mu_{0}(\vec{H}+\vec{M})=\mu_{0}(\vec{H}+\chi \vec{H})=\mu_{0} \mu_{r} \vec{H}
$$

where $\mu_{0}$ is the magnetic permeability of space, $\mu_{r}$ is the relative permeability of the magnetic material, $\chi$ is the susceptibility, and $\mathbf{M}$ the internal magnetization. The relative permeability is large for ferromagnetic materials such as iron and nickel, and these are the materials most commonly used in magnetic microactuators.

There are two classes of ferromagnets: hard magnets, which retain some of their magnetic polarization even after an applied external magnetic field is removed, and soft magnets, which exhibit internal magnetization only in the presence of an external magnetic field. In general, higher magnetization levels are available in hard magnetic materials. Shape anisotropy, more so than the orientation to the induction field, determines the direction of polarization in the material. A rod-shaped piece of material, for example, will usually have an internal field pointing in the longitudinal direction, and a thin plate will usually exhibit magnetization in the plane of the plate, even when the induction field is oriented in another direction. This is a particular consideration for soft-magnetic materials-- hard magnetic materials can be magnetized before being cut into the desired shape, allowing the designer control over the orientation of the magnetization relative to the shape.

In the presence of an external magnetic field, both hard and soft magnets will rotate until their internal magnetization is parallel to the local external magnetic field lines. A net force for translational motion, however, is only exerted in the case of non-uniform magnetic field. The equations for torque and force are given by (Yesin et al., 2006):

$$
\begin{array}{r}
\vec{T}=V \vec{M} \times \vec{B} \\
\vec{F}=V(\vec{M} \bullet \nabla) \vec{B}
\end{array}
$$

where $V$ is the volume of the slug of ferromagnetic material. The force will draw the slug in the direction of increasing field intensity, or towards the magnet.

The challenge for magnetic actuation is not getting the robot to move, but rather getting it to move in a controllable fashion. Combining the distance dependencies of the magnetization and of the gradient of the magnetic field, the force for a soft magnet is inversely proportional to the fifth power of the distance between the robot and the electromagnet coil (Yesin et al., 2006). For a hard magnet, it depends only on the inverse of the cube. Once the robot begins to move, it quickly accelerates towards the electromagnet, and without careful control it will snap to the electromagnet. This problem is addressed in part by sizing and placing the electromagnets so that their interior volume is much greater than the volume in which the robot is meant to operate. This reduces the variation in the field strength over the playing field area and allows the opposing electromagnet to counter one electromagnet's force at a reasonable current level. The non-linearity is also addressed by using oscillatory motion or multiple coils operating simultaneously to build a linear field, such as the Helmholtz or Maxwell coil configurations, which are common in the magnetic resonance imaging (MRI) industry (Yesin et al., 2006). 


\subsection{Ferromagnetic-Core-Based Microrobots}

Floyd et al. use a hard magnet as their robotic element (Floyd et al., 2008; Pawashe, 2008; Pawashe, 2009, a; Pawashe, 2009, b). The robot is cut from a magnetized sheet of NdFeB using laser machining. The robot, shown in motion in Fig. 7, is chevron-shaped, $100 \mu \mathrm{m}$ thick, and approximately $250 \mu \mathrm{m}$ by $130 \mu \mathrm{m}$ in the $x y$-plane. It has a mass of $25.6 \mu \mathrm{g}$ and a magnetization of $500 \mathrm{kA} / \mathrm{m}$, and it operates within a cubic workspace approximately $2 \mathrm{~cm}$ on a side with gradient fields as strong as $149 \mathrm{mT} / \mathrm{m}$. They use short duration, periodic fluctuations in the magnetic field to control robot motion.

The robot body is controlled using five independent electromagnetic coils. Four of these are in the plane of the robot's playing field and the fifth is below the playing field. Two actuation techniques can be employed, and both of them take advantage of stick-slip motion of the robotic mass induced by pulsed magnetic fields.

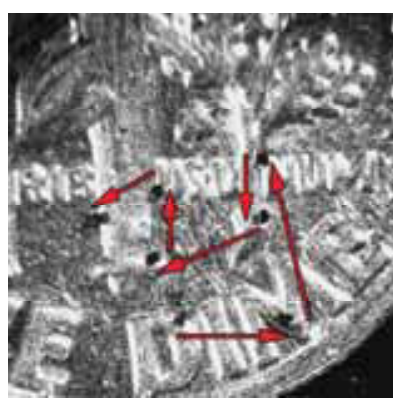

Fig. 7. The Carnegie Mellon Mag- $\mu$ Bot in motion, by Floyd and Pawashe at Carnegie Mellon University. The robot is approximately $300 \mu \mathrm{m} \times 300 \mu \mathrm{m} \times 100 \mu \mathrm{m}$ (Floyd, 2008; Pawashe, 2008; Pawashe, 2009, a; Pawashe, 2009, b). Figure used with permission from the authors.

The first technique, In-Plane Pulsing (IPP), first uses the fifth coil to clamp the robot to the playing field and then orients the robot using the other four coils. Then, with the clamping coil still in effect, translation is effected by applying a saw tooth magnetic field waveform with the four in plane coils. The second technique, Out-of-Plane Pulsing (OPP), reverses the role of the clamping coil and the four in-plane coils. The coil beneath the work surface is pulsed to create a saw tooth magnetic field, and the in-plane coils are held constant with similar stick-slip translational motion as a result. Experimental results show that robot velocities increase with increased pulsing frequencies for both IPP and OPP methods; however IPP produces more consistent results fitting an exponential attack curve. OPP produces higher velocities, on the order of $2.8 \mathrm{~mm} / \mathrm{s}$, where the maximum velocity for IPP was only $700 \mu \mathrm{m} / \mathrm{s}$. The authors suggest OPP for coarser motion and IPP for finer control.

While this robot is tailored to surface motion, several groups have pursued similar strategies for microrobots tailored to biomedical applications. The group of S. Martel at Ecole Polytechnique de Montreal has adapted a clinical magnetic resonance imaging (MRI) platform for control of a ferromagnetic bead within a fluid environment, and have demonstrated their work in vivo in swine (Mathieu et al., 2006; Martel, 2007; Tamaz et al., 2008). Yesin et al. at ETH Zurich have developed a nickel, football-shaped microrobot that 
could be used for microsurgery in the human eye as well as cardiovascular applications (Yesin et al., 2006).

ETH Zurich has also developed "nanohelices" or tiny spiral structures (about $60 \mu \mathrm{m}$ in length) that have a soft magnetic head which allows them to be manipulated by a magnetic field (Kratochvil et al., 2008). With these structures they have demonstrated controlled conversion between rotary and linear motion, which finds application both as a biomimetic swimming microrobot actuator (like a bacteria flagellum) and as an instrumentation tool for rotating structures within a scanning electron microscope. Similar work at a larger size-scale (about $1 \mathrm{~mm}$ in length) using a spiral of tungsten wire has been demonstrated by Yamazaki et al. (Yamazaki et al., 2004). Biomimetic, bacteria-flagella-type motion has also been demonstrated by Dreyfus et al. (Dreyfus et al., 2005), who formed their actuator from a string of magnetic beads held together by DNA and attached to a red blood cell.

\subsection{Resonant Magnetic Robots}

The resonant magnetic robots, or "Magmites" developed by the group at ETH Zurich (Vollmers et al., 2008; Kratochvil et al., 2009; Frutiger et al., 2008; Frutiger et al., 2009) consist of a base frame that contains a spring and two ferromagnetic masses. One mass is attached to the frame and the other to the spring. The entire robot covers an area of $300 \mu \mathrm{m} \times 300 \mu \mathrm{m}$ and is about $150 \mu \mathrm{m}$ thick. In a spatially uniform magnetic field, the two adjacent pieces of ferromagnetic material magnetize and experience interaction forces but no net force. When the field is oscillated at the appropriate frequency, it excites the mechanical resonance of the structure, swinging the mass attached to the spring (the hammer) within the plane of the frame. By applying a clamping field at the right moment in the cycle, the momentum of this hammer action is transformed into translational motion. The direction of motion depends on the phase difference between the magnetic excitation field and the clamping field. Clamping is accomplished electrostatically using the playing field electrode array. Unidirectional motion is even possible in the absence of the clamping field due to the non-uniformity of the friction forces during the resonator cycle.

Magmites are fabricated through a surface micromachining process that utilizes copper or gold for the frame and spring structure and electroplated nickel for the soft magnetic masses, as is illustrated in Fig. 8. Dimples on the bottom of the frame are used to reduce the contact area with the substrate. Magmites have been shown to operate in an environment of up to $60 \%$ relative humidity. They use fields as low as $2 \mathrm{mT}$ operating in the frequency range of $1 \mathrm{kHz}$ to $5 \mathrm{kHz}$. In the 2009 competition in Graz, Austria, they set the record for the $2 \mathrm{~mm}$ dash with a time of $0.326 \mathrm{~s}$ (a speed of $6.1 \mathrm{~m} / \mathrm{s}$, faster speeds were demonstrated informally). Multirobot control is demonstrated by using robots engineered for different resonance frequencies (Kratochvil et al., 2009). 

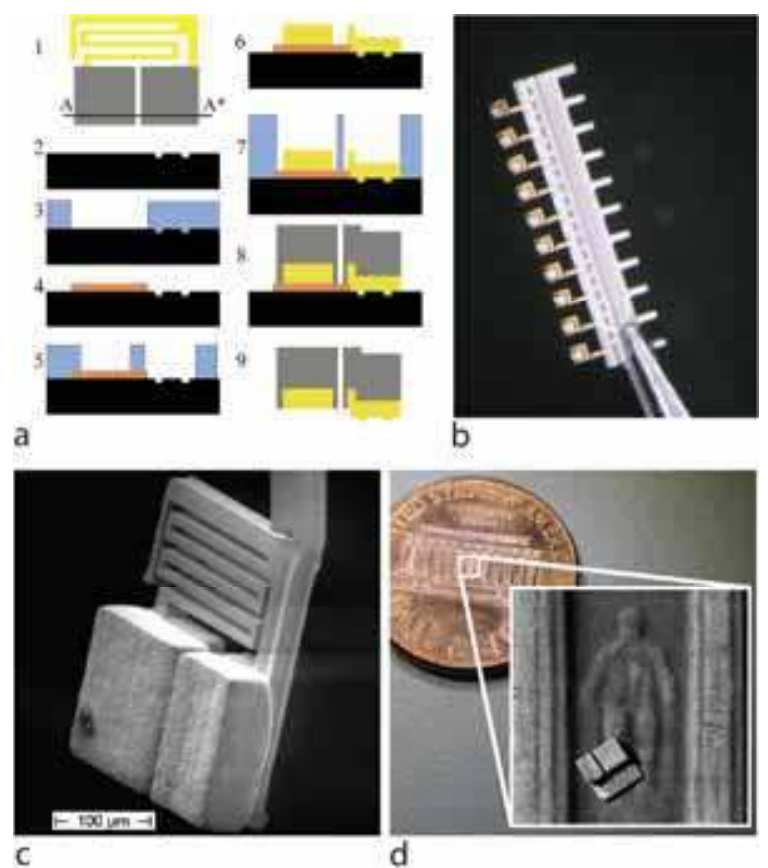

Fig. 8. a) The fabrication procedure for first-generation magmites uses surface micromachining with electroplated gold and nickel layers; b) A strip of released Magmites still attached to a tether for handling; c) a scanning electron micrograph of a Magmite; d) the Magmite on an American penny, reprinted from (Frutiger, 2009). Figures by Frutiger, Vollmers and Kratochvil and used with permission by the authors.

\subsection{Magnetic Microrobot Challenges}

Fabrication limitations pose a significant challenge for this class of microrobots. Traditional silicon micromachining incorporates few materials which are ferromagnetic. Only Nickel is available in a commercial microfabrication process, and, with a relative permeability of only 600 , it is far less ferromagnetic than materials such as permalloy used in common macroscale magnetic systems. Custom electroplating can be used to integrate a handful of other materials, but the best magnetic materials are still not available through these methods (Cugat et al., 2003). Techniques such as laser cutting allow for greater material breadth, but it is difficult to then integrate the magnetic material with other microstructures.

Multirobot cooperation also presents a challenge, particularly for ferromagnetic-core-based robots, as there is no way to selectively address a particular robot with the magnetic field, alone. However, Pawashe et al. (Pawashe et al., 2009) have used an underlying electrode array that uses localized electrostatic clamping to differentiate between robots. For resonant microrobots, individual robots can be targeted by their resonant frequencies.

Even with these challenges, magnetic microrobots have outperformed the scratch drive actuator-based robots thus far in the Nanogram soccer competitions. It seems likely that 
fabrication methods will expand to incorporate more magnetic material variety if there is sufficient need. Furthermore, given the prevalence of magnetic resonance imaging (MRI) systems in medicine, magnetic microrobots are an enticing candidate for medical applications.

\section{Other Microrobot Actuation Methods}

While only electrostatic and magnetic actuators have been used by teams competing in the Nanogram events, there are a variety of other actuation methods that have been used for microrobotics. In particular, thermally actuated microrobotics has been demonstrated by several groups (Ohmichi et al., 1997; Ebefors et al., 2000; Kladitis et al., 2000; Baglio et al., 2002; Sul et al., 2006; Brown et al., 2007). Several groups have also investigated robots powered by external vibrations (Yasuda et al., 1994; Yasuda et al., 1995; Saitou et al., 2000), and piezoelectric actuators (Smits, 1989; Wood et al., 2003; Nguyen and Martel, 2006; Edqvist, 2009). Some of these systems are not microrobot systems in the sense of a microscale independent actor, but rather micro- or nano-positioning systems where the actuators are fixed to a substrate and are used to manipulate micro-sized components, like a microscale conveyer belt. In the fluid domain, a few groups have even been working with bacteria flagella-based propulsion (Behkam and Sitti, 2007; Martel et al., 2008) and semiconductor diode propulsion (Chang et al., 2007). These technologies are not as easily adapted to a surface walking application, but are briefly reviewed as they relate to the larger nanorobotic goals of minimally invasive surgery, microassembly, and micropositioning.

\subsection{Thermal Actuation}

Thermal actuation is prevalent in microsystems, and so, while there has as yet been no thermally actuated competitor in the Nanogram events, the future appearance of a robot of this type seems likely. These robots rely on thermal expansion for motion. There are two large classes of thermally actuated robots: "inchworm" drives and "impulse" or "impact" drives. In an inchworm drive cyclic deformation results in forward motion in a manner that is relatively independent of the time scale of the deformation. In such devices the speed should simply be linearly dependent on the excitation frequency. In contrast, for an "impulse" drive momentum generation is essential to the forward motion, making the actuation method dependent on the time scale of the excitation. In other words, the inchworm drive is a sort of shuffle step, while the impulse drive requires an initial sharp kick. (It's the Charleston of actuation mechanisms.)

\subsubsection{The physics of thermal actuation}

Thermal actuators are based on thermal expansion. Most materials expand when heated, including most of the materials common in micromachining. Thermal actuators can be divided into three broad classes: thermal bimorphs, single material structures, and multiphase devices. The final category, which includes ink jet heads where the expansion of a gas bubble is used to force a fixed volume of ink out of a small cavity, has not been applied to microrobotics. 
Thermal bimorphs consist of layered structures containing materials with different thermal expansion properties. When heated, the structures will bend away from the side of the structure containing the material with the higher expansion coefficient. Because of fabrication limitations, thermal bimorphs are mostly used for out-of-plane motion. In contrast, single-material thermal actuators are more commonly used for in-plane motion, although they can be used for both in-plane and out-of-plane motion. Single-material actuators sometimes rely on localized heating to create motion. This is certainly the case for laser-heated structures, but also can occur in Joule-heated devices if the device geometry confines the heating to a small area by creating an area that is thermally confined. An example of this is shown on the left of Fig. 9.
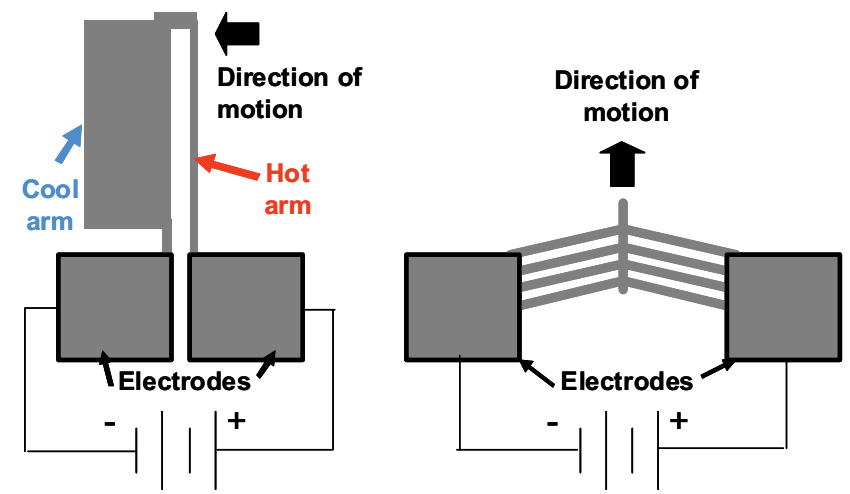

Fig. 9. Illustration of two common types of single-material thermal actuators. On the left, the geometry of the structure results in differential heating causing the thin arm to expand and bend the structure towards the thick arm. In a bent beam actuator like on the right, the beams are oriented so that thermal expansion leads to forward motion of the shuttle structure in the middle.

Another common type of thermal actuator is a "bent-beam" actuator, illustrated on the right of Fig. 9. In these chevron-shaped structures, current is passed through the beam causing heating. Because of the bend in the center of the beam, expansion leads to translational motion in-plane in the direction of the chevron's base.

While much of the interest in thermally actuated robotic systems has been directed at miniature robots (Kladitis and Bright, 2000, Ebefors et al., 2000), a number of groups have demonstrated impulse-drive microrobots that are actuated through local heating by lasersan idea that was introduced by (Baglio et al., 2002). Sul et al. at UNC Chapel Hill have illustrated an elegant tripod shaped robot that is actuated by local heating (Sul et al., 2006). The three legs of the robot are metal-film bimorphs. At equilibrium the legs arch down to the substrate because of residual stress from the fabrication process. When heated, the legs further deflect due to differential thermal expansion coefficients in the two materials. The rapid heating of one leg leads to a stepwise transition on a low-friction surface. There are two phases to the motion, the contraction phase, in which there is rapid motion of the device which breaks the adhesive contact and overcomes sliding friction for the contacts, and the relaxation phase where the device returns to its original shape. In the contraction phase for 
the UNC microrobot, only one leg is heated, but, in the relaxation phase, the heat has spread throughout the device and dissipates in a more uniform fashion, causing the device to move away from the leg that was heated (Sul et al., 2006). Thus the device can be steered by focusing the laser on the appropriate leg.

A similar micro-impact drive mechanism was described by Ohmichi et al. on the millimeter scale (Ohmichi et al., 1997). These robots were fabricated from aluminum alloy by precision cutting techniques. Like the UNC microrobot, these structures relied on a fast initial stage where the motion is generated, followed by a slow relaxation period. The Ohmichi robot consisted of a main body that is separated from a weight block by a narrow neck. A laser was directed onto the neck causing rapid thermal expansion which pushed the weight away from the body, resulting in an impulsive force that exceeded the static friction force. The heat then dissipated throughout the structure causing a slower relaxation to the original shape that does not counteract the initial motion. With repeated optical pulsing (up to 5 $\mathrm{kHz}$ ) the team achieved speeds of up to $31 \mathrm{~mm} / \mathrm{s}$. The robot was approximately $1.7 \mathrm{~mm} \mathrm{x}$ $0.6 \mathrm{~mm} \times 0.4 \mathrm{~mm}$.

One challenge for these types of robots is the requirement for optical access and fine control of the optical system. A further challenge for these systems is that the complexity of the control system would significantly increase for multirobot cooperation. Finally, these systems have relied on special low-friction surfaces.

Friction forces are exploited by Brown et al. at Dalhousie University (Brown et al., 2007). Their "frictional crawler" consists of 3 "feet" linked by two actuators. The locomotion mechanism uses a shuffle step that takes advantage of the differences in contact area that result from coupling one or two feet to achieve net translational motion. The team used bent-beam actuators, of the type illustrated in Fig. 9 (right). Electrical connection to the actuators was accomplished through rails on the substrate. The structures were fabricated using a silicon-on-insulator process by the Micragem process $^{1}$ (CMC Microsystems, 2009) that results in a single-crystal-silicon actuators. The overall device dimensions were $1400 \mu \mathrm{m}$ $X 525 \mu \mathrm{m} \times 10 \mu \mathrm{m}$. The actuators required $2.75 \mathrm{~V}$ for operation. At this voltage, each drew about $69 \mathrm{~mA}$. The device traveled at $0.7 \mathrm{~mm} / \mathrm{s}$ at its maximum frequency of $300 \mathrm{~Hz}$ (the limit for the thermal actuators) and was observed to develop a horizontal force greater than $130 \mu \mathrm{N}$. Significantly, it operated reliably even when carrying a load over 100 times its own weight of $1 \mu \mathrm{N}$. Good contact between the device and the rails was essential to operation, and the team used a thin film of electrically conductive grease on the rails to maintain that contact in the face of device imperfections. The rails, of course, limit the range and turning capability of this robot, but the overall locomotion method is quite interesting.

\footnotetext{
${ }^{1}$ Certain commercial equipment, instruments, or materials are identified in this review to adequately specify the experimental procedure. Such identification does not imply recommendation or endorsement by the National Institute of Standards and Technology, nor does it imply that the materials or equipment identified are necessarily the best available for the purpose.
} 


\subsection{Vibration Actuation}

Vibration was used for microrobot locomotion by Yasuda et al., who have built a millimeterscale walking robot (Yasuda et al., 1994; Yasuda et al., 1995). Their robot had six legs: four of which supported the body and transmitted the vibration energy to the robot, and two of which were free to "kick" the base to direct its motion. The kicking legs were cantilevered massspring units designed for particular resonant frequencies. One of the kicking legs caused right turns and the other left turns. The resonant frequency for the kicking legs was well below that of the four support legs. By changing the spectra of the vibration field, one, both, or neither of the legs can be activated allowing for turning, straight motion, and a full stop. The microrobot measured $1.5 \mathrm{~mm}$ by $0.7 \mathrm{~mm}$. It was fabricated by surface micromachining with a sacrificial layer of phosphosilicate glass (PSG) and structural layers of polysilicon and polyimide (used to create hinges). Following fabrication, the final structure was created by folding using micro probes. The kicking legs were tailored to resonance frequencies in the $100 \mathrm{~Hz}$ to $1000 \mathrm{~Hz}$ range, and motion of up to $7 \mathrm{~mm} / \mathrm{s}$ was observed.

Saitou et al. use microcantilever impactors designed for specific mechanical resonance frequencies in their micropositioning system (Saitou, 2000). The actuators are anchored to the substrate, and they control a shuttle intended for fine positioning of a generic microcomponent. The actuators are suspended masses that resonate in the plane of the substrate. As they resonate they impact against the side of the shuttle structure moving it forward or backward depending on the relative orientation of the actuator and the shuttle. Two actuators with one resonance frequency are positioned to cause forward motion, and another two actuators with a distinct resonance frequency are positioned to cause reverse motion. When the entire system is exposed to a vibration field, the frequency of that vibration determines the direction of motion of the shuttle. The system was fabricated using the PolyMUMPS process, which is a multi-user, polysilicon-based, surface micromachining process (MEMSCAP, 2009). The resonance frequencies used for the structure are in the range of 1 $\mathrm{kHz}$ to $10 \mathrm{kHz}$, and result in motion at the speed of $2 \mathrm{~mm} / \mathrm{s}$ to $5 \mathrm{~mm} / \mathrm{s}$.

While robots with this vibration actuator scheme have not yet been used in the Nanogram events, the vibration actuation mechanism offers many of the same advantages as the Magmite resonant magnetic actuation method. The use of resonance allows for multirobot cooperation by allowing individual robots to be targeted with a global energy field through frequency control.

\subsection{Piezoelectric Actuation}

The presence of an electric field induces stress in piezoelectric materials. This property has been exploited to create a variety of electrically powered actuators. A few groups have used piezoelectric actuators for millimeter-to-centimeter scale robots, including flying insectmodeled miniature robots (Wood et al., 2003) and vibratory walking miniature robots (Nguyen and Martel, 2006, Edqvist et al., 2009). While these existing systems are a one to two orders of magnitude larger than microrobots, the scaling limits of the piezoelectric actuation methodhave not yet been fully explored.

Piezoelectricity was first identified in quartz crystals and is widely applied in sonar systems and in quartz crystal oscillators. Material processing presents a significant challenge to the 
development of piezoelectric MEMS. Techniques that are commonly used for preparing bulk piezoelectric materials are not suited for microfabrication, and the piezoelectric coupling coefficients for thin-film materials are often significantly lower than their bulk counterparts (Liu, 2006). However, recent advances in deposition techniques for PZT (lead zirconate titanate) (Wang, 2003) and PVDF (polyvinylidenfluoride, a piezoelectric polymer) (Manohara et al., 1999) have increased the prevalence of this transduction mechanism.

\subsection{Biological and Other Actuation Methods}

The work of several groups in which ferromagnetic core based robots were used to form flagella-like propulsion structures was reviewed in the magnetic actuation section (Kratochvil et al., 2008; Yamazaki et al., 2004; Dreyfus et al, 2005). Other groups, however, work directly with biological structures for micron-scale aqueous propulsion. Martel et al. have worked with "Magnetotactic Bacteria (MTB)," which is bacteria that contains magnetosomes-membrane-based nanoparticles of a magnetic iron that respond to magnetic fields (Martel et al., 2008). They have attached these flagellated bacteria to microbeads and demonstrated controlled motion using a magnetic resonance imaging (MRI) system. Behkam and Sitti use chemicals instead of magnetic fields to control their bacterial flagella actuators, which use Serratia marcescens bacteria attached to polystyrene beads (Behkam \& Sitti, 2007). Other mechanisms for micro- and nano-scale actuation in fluids have been described in the literature that are beyond the scope of this work. Many are reviewed by Chang et al., who also describe a novel diode-based actuation method for motion in fluids (Chang et al., 2007). These systems rely on a fluid environment and are not suitable for the surface crawling application of microsoccer. However, these systems may some day find a use in medical applications.

\section{Multirobot Cooperation}

Recent work by Bretl (Bretl, 2007), Donald et al. (Donald et al., 2008), and Kratochovil et al. (Kratochvil et al., 2009) has also investigated control of multiple scratch drive actuators moving on the same substrate. In the case of (Donald et al., 2008) the authors take advantage of a group of individual scratch drive actuators with slightly different properties (arm lengths, etc.) that respond to various motion primitives (waveforms generated by altering the period and amplitude) differently. The preimage planning mentioned earlier takes this into account.

Bretl's work (Bretl, 2007) explores the possible control of multiple identical robots that are exposed to the same voltage control sequence. He shows how two robots with unicycle dynamics and a bounded turning radius can be controlled to an arbitrary point from arbitrary positions.

Multi-robot control for magnetic devices has also been demonstrated by Floyd et al. (Floyd et al., 2008), by utilizing electrostatic clamping of the playing field in addition to the pulsed magnetic field actuation. In subsequent work by Pawashe (Paswashe, 2009), the playing field is composed of an array of independent interdigitated electrodes. In other words, the playing field is composed of $m \times n$ subfields similar to those used for actuation of scratch drive actuators. For purpose of demonstration, a two by two array was created. By applying a voltage field to an individual field, any robotic device on that subfield is held static by 
electrostatic clamping. In this manner, a particular device can be held still while other devices are controlled by the pulsing magnetic fields.

Kratochvil et al. (Kratochvil et al., 2009) demonstrated multirobot movement by building two different microrobot devices that are sensitive to different resonant frequencies. By varying the frequency of the oscillating magnetic field, either one or both of the devices will move. Their work is illustrated in Fig. 10.
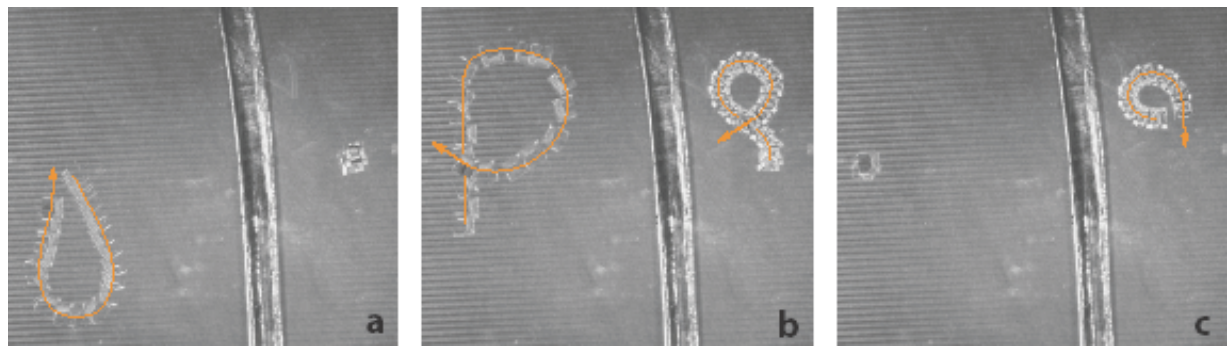

Fig. 10. Multiple robots driving on the same substrate (Kratochvil et al., 2009). The vertical line is a physical barrier to help prevent robot collisions and expedite experiments. In a and c, one robot is moving while the other is stationary. In b, the two robots are moving in different patterns. Figures are by Frutiger, Vollmers and Kratochvil and are used with permission by the authors.

\section{Conclusion}

After only three years, the RoboCup Nanogram events have produced a variety of creative microrobot systems. Competitors so far only used magnetic and electrostatic actuation, but other actuation technologies may emerge. Thermal, vibratory, and piezoelectric actuation all offer potential performance advantages. As a step down the road to a revolution in minimally invasive surgery and micromanufacturing, these small robots might indeed have a big impact.

\section{References}

Abbott, J ; Nagy, Z. ; Beyeler, F \& Nelson, B. (2007). Robotics in the small. IEEE Robotics and Automation Magazine, Vol. 14, No. 2, pp. 92-103.

Akiyama, T. \& Shono, K. (1993). Controlled stepwise motion in polysilicon microstructures. Journal of Microelectromechanical Systems, Vol. 2, pp. 106-110.

Baglio, S. ; Castorina, S. ; Fortuna, L. \& Savalli, N. (2002). Novel microactuators based on photo-thermo-mechanical actuation strategy. Proceedings of IEEE Sensors 2002, Orlando, Florida, pp. 192-197.

Behkam, B. \& Sitti, M. (2007). Bacterial flagella-based propulsion and on/off motion control of microscale objects. Applied Physics Letters, Vol. 90, 023902.

Bretl, T. (2007). Control of many objects using few instructions. Proceedings of Robotics : Science and Systems, Atlanta, Georgia, July 2007.

Brown, M. ; Hubbard, T. \& Kujath, M.. (2007). Development of a long-range untethered frictional microcrawler. Journal of Micromechanics and Microengineering, Vol. 17, pp. 1025-1033.

CMC Microsystems, http:/ / www.cmc.ca/index.htm, site accessed Aug. 17, 2009. 
Chang, S. T. ; Paunov, V. N. ; Petsey, D. N. \& Velev, O. D. (2007). Remotely powered selfpropelling particles and micropumps based on miniature diodes. Nature Materials, Vol. 6, March 2007, pp. 235-240.

Cugat, O.; Delamare, J. \& Reyne, G. (2003). Magnetic micro-actuators and systems (MAGMAS). IEEE Transactions on Magnetics, Vol. 39, No. 5, pp. 3607-3612.

Dario, P. ; Velleggi, R. ; Carrozza, M. C. ; Montesi, M. C. \& Cocco, M. (1992). Microactuators for microrobots: a critical survey. Journal of Micromechanics and Microengineering, Vol. 2, pp. 141-157.

Donald, B. ; Levey, C. ; McGray, C. ; Rus, D. \& Sinclair, M. (2003). Power delivery and locomotion of untethered microactuators. Journal of Microelectromechanical Systems, Vol. 12, No. 6, pp. 947-959.

Donald, B.; Levey, C.; McGray, C.; Paprotny, I. \& Rus D. (2006). An untethered, electrostatic, globally controllable MEMS micro-robot. Journal of Microelectromechanical Systems, Vol. 15, No. 1, pp. 1-15.

Donald, B. ; Levey, C. \& Paprotny, I. (2008). Planar microassembly by parallel actuation of MEMS microrobots, Journal of Microelectromechanical Systems, Vol. 17, No. 4, pp. 789-808.

Dreyfus, R. ; Baudry, J. ; Roper, M. L. ; Fermigier, M. ; Stone, H. A. ; and Bibette, J. (2005). Microscopic artificial swimmers. Nature, Vol. 437, pp. 862-865.

Dubin, L. E. (1957). On curves of minimal length with a constraint on average curvature and with prescribed initial an dterminal positions and tangents. American Journal of Mathematics, Vol. 79, No. 3, pp. 497-516.

Ebefors, T. ; Mattsson, J. U. ; Kalvesten, E. \& Stemme, G. (2000). A robust micro conveyer realized by arrayed polyimide joint actuators, Journal of Micromechanics and Microengineering, Vol. 10, pp. 337-349.

Edqvist, E. ; Snis, N. ; Casanova Mohr, R. ; Scholz, O. ; Corradi, P. ; Gao, J. ; Dieguez, A. ; Wyrsch, N. \& Johannson, S. (2009). Evaluation of building technology for mass producible millimeter-sized robots using flexible printed circuit boards. Journal of Microelectromechanics and Microengineering, Vol. 19, 075011.

Fan, L. ; Tai, Y. \& Muller, R. (1988). Integrated movable micromechanical structures for sensors and actuators. IEEE Transactions on Electron Devices, Vol. 35, No. 6, pp. 724-730.

Firebaugh, S. L. \& Piepmeier, J. A. (2008). The RoboCup Nanogram League : an opportunity for problem-based undergraduate education in microsystems. IEEE Transactions on Education, Vol. 51, No. 3, pp. 394-399.

Floyd, S. ; Pawashe, C. \& Sitti, M. (2008). An untethered magnetically actuated micro-robot capable of motion on arbitrary surfaces. Proceedings of the IEEE International Conference on Robotics and Automation, pp. 419-424, Pasadena, CA, May 19-23, 2008.

a Frutiger, D. R. ; Vollmers, K. ; Kratochvil, B. E. \& Nelson, B. J. (2008). Small, fast and under control : wireless resonant magnetic micro-agents. Proceedings of the International Symposium on Experimental Robotics, July 2008.

b Frutiger, D. R. ; Kratochvil, B. E. ; Vollmers, K. \& Nelson, B. J. (2008). Magmites - wireless resonant magnetic microrobots. Proceedings of the IEEE International Conference on Robotics and Automation, pp. 1770-1771, Pasadena, CA, May 19-23, 2008.

Frutiger, D. R. ; Vollmers, K. ; Kratochvil, B. E. \& Nelson, B. J. (2009). Small, fast and under control: wireless resonant magnetic micro-agents. International Journal of Robotics Research, November, 2009.

Gates, B. (2007). A robot in every home. Scientific American, Jan., pp. 58-65. 
Gauthier, M. \& Piat, E. (2004). An electromagnetic micromanipulation system for single cell manipulation. Journal of Micromechatronics, Vol. 2, No. 2, pp. 87-119.

Hayt, Jr., W. H. \& Buck, J. A. (2006). Engineering Electromagnetics, 7th Ed. McGraw-Hill Higher Education, ISBN 0-07-252495-2, New York, NY.

Hermes, H. (1974). On local and global controllability. SIAM J. Contr., vol. 12, pp. 252-261.

Huikai, X. ; Erdmann, L. ; Zhu, X. ; Gabriel, K. \& Fedder, G. (2002). Post-CMOS processing for high-aspect-ratio integrated silicon microstructures. Journal of Microelectromechanical Systems, Vol. 11, No. 2, pp. 93-101.

Kladitis, P. E. \& Bright, V. M. (2000). Prototype microrobots for micro-positioning and micro-unmanned vehicles. Sensors and Actuators, Vol. 80, pp. 132-137.

Kovacs, G. (1998). Micromachined Transducers Sourcebook, pp. 277-289, WCB McGraw-Hill, ISBN 0-07-290722-3, Boston.

Kratochvil, B. E. ; Dong, L. ; Zhang, L. ; Abbott, J. J. and Nelson, B. J. (2008). Nanohelices as motion converters. Proceedings of the 2008 IEEE/RSJ International Conference on Intelligent Robots and Systems, Nice, France, Sept. 22-26, 2008.

Kratochvil, B. E. ; Frutiger, D. ; Vollmers, K. \& Nelson, B. J. (2009). Visual servoing and characterization of resonant magnetic actuators for decoupled locomotion of multiple untethered mobile microrobots, Proceedings of the IEEE International Conference on Robotics and Automation, pp. 1010-1015, Kobe, Japan, May 12-17, 2009.

Lal, A.; Duggirala, R. \& Li, H. (2005). Pervasive power: a radioisotope-powered piezoelectric generator. IEEE Pervasive Computing, Vol. 4, No. 1, pp. 53-61.

Lionis, G \& Kyriakopoulos, K. J. (2007). PWM control for a micro-robot moving on a discrete curvature trajectory set. Proceedings of the 2007 IEEE International Conference on Robotics and Automation, pp. 2324-2329, Rome, Italy, April 10-14, 2007.

Liu, C. (2006). Foundations of MEMS, Pearson Education, Inc., ISBN 0-13-147286-0, Upper Saddle River, New Jersey.

Lozano-Perez, T. ; Mason, M. T. \& Taylor, R. H. (1984). Automatic synthesis of fine-motion strategies for robots. International Journal of Robotics Research, Vol. 3, No. 1, pp. 3-24.

Madou, M. (1997). Fundamentals of Microfabrication, CRC Press, ISBN 0-8493-0826-7, Boca Raton, FL.

Manohara M. ; Morikawa, E.; Choi, J. \& Sprunger, P.T. (1999). Transfer by direct photo etching of poly(vinylidene fluoride) using x-rays. Journal of Microelectromechanical Systems, Vol. 8, pp. 417-422.

Markus, K. ; Koester, D. ; Cowen, A. ; Mahadevan, R. ; Dhuler, V. ; Roberson, D. \& Smith, L. (1995). MEMS infrastructure: the multi-user MEMS processes (MUMPS). Proceedings of the SPIE, Vol. 2639, pp. 54-63.

aMartel, S. (2005). Special surface for power delivery to wireless micro-electro-mechanical systems. Journal of Micromechanics and Microengineering, Vol. 15, pp. S251-S258.

bMartel, S. (2005). Fundamental principles and issues of high-speed piezoactuated threelegged motion for miniature robots designed for nanometer-scale operations. The International Journal of Robotics Research, Vol. 24, No. 7, pp. 575-588.

Martel, S. (2007). Magnetic resonance propulsion, control and tracking at $24 \mathrm{~Hz}$ of an untethered device in the carotid artery of a living animal : an important step in the development of medical micro- and nanorobots. Proceedings of the 295h Annual International Conference of the IEEE EMBS Cite Internationale, pp. 1475-1478, Lyon France, August 23-26, 2007. 
Martel, S.; Felfoul, O. \& Mohammadi, M. (2008). Flagellated bacterial nanorobots for medical interventions in the human body. Proceedings of the $2^{\text {nd }}$ Biennial IEEE/RASEMBS International Conference on Biomedical Robotics and Biomechatronics, pp. 264-269, Scottsdale, Arizona, Oct. 19-22.

Mathieu, J.-B. ; Beaudoin, G. \& Martel, S. (2006). Method of propulsion of a ferromagnetic core in the cardiovascular system through magnetic gradients generated by an MRI system. IEEE Transactions on Biomedical Engineering, Vol. 53, pp. 292-299.

MEMSCAP, Inc., http://www.memscap.com/index.php, site accessed Aug. 17, 2009.

Nathanson, H. ; Newell, W., Wickstrom, R. \& Davis, J. (1978). The resonant gate transistor. IEEE Transactions on Electron Devices, Vol. 14, pp. 117-133.

Nguyen, A. T. \& Martel, S. (2006). Miniaturization of a piezo-actuation system embedded in an instrumented autonomous robot. Proceedings of the 4th International IEEENEWCAS Conference, pp. 261-264, Gatineau, Quebec.

Ohmichi, O. ; Yamagata, Y. \& Higuchi, T. (2002). Micro-impact drive mechanisms using optically excited thermal expansion. Journal of Microelectromechanical Systems, Vol. 6, No. 3, pp. 200-207.

Patil, A. ; Patil, V. ; Shin, D. W. ; Choi, J. ; Paik, D. \& Yoon, S. (2008). Issues and challenges facing rechargeable thin film lithium batteries. Materials Research Bulletin, Vol. 43, pp. 1913-1942,

Pawashe, C. ; Floyd, S. \& Sitti, M. (2008). Dynamic modelling of stick slip motion in an untethered magnetic micro-robot. Proceedings of Robotics: Science and Systems IV, Zurich, Switzerland, June 2008.

aPawashe, C. ; Floyd, S. \& Sitti, M. (2009). Modeling and experimental characterization of an untethered magnetic micro-robot. The International Journal of Robotics Research, Vol. 28, pp. 1077-1094.

bPawashe, C.; Floyd, S. \& Sitti, M. (2009). Multiple magnetic microrobot control using electrostatic clamping. Applied Physics Letters, Vol. 94, 164108.

Petersen, K. (1978). Dynamic micromechanics on silicon: techniques and devices. IEEE Transactions on Electron Devices, Vol. 25, No. 10, pp. 1241-1250.

Piepmeier, J. A. \& Firebaugh, S. L. (2008). Vision-based control of a scratch drive microrobot. Proceedings of the Southeastern Symposium on System Theory, pp. 352-355, New Orleans, Louisiana.

Piepmeier, J. A. \& Firebaugh, S. L. (2010). PWM control accuracy for scratch drive actuators, submitted to the IEEE International Conference on Robotics and Automation, Anchorage, Alaska, May 2010.

Ross, R. (2007). Robotic fly takes off. Technology Review, http://www.technology review.com/Infotech/19068/, created July 19, 2007, accessed Aug. 12, 2009.

Saitou, K. ; Wang, D.-A. \& Wou, S. J. (2000). Externally resonated linear microvibromotor for microassembly. Journal of Microelectromechanical Systems, Vol. 9, No. XX, pp. 336-346.

Sievers, T . \& Fatikow, S. (2005). Visual servoing of a mobile microrobot inside a scanning electron microscope. Proceedings of the IEEE/RSJ International Conference on Intelligent Robots and Systems, pp. 1350-1354, Edmonton, Alberta, March 2008.

Sitti, M. (2007). Microscale and nanoscale robotics systems. IEEE Robotics \& Automation Magazine, Vol. 14, No. 1, pp. 53-60. 
Smits, J. G. (1989). Is micromechanics becoming a new subject for academic courses or the design of a piezoelectric on silicon microrobot, Proceedings of the 8th University/Government/Industry Microelectronics Symposium, pp. 141-145, Westborough, MA. 1989.

Sniegowski, J. \& Garcia, E. (1996). Surface-micromachined gear trains driven by an on-chip electrostatic microengine. IEEE Electron Device Letters, Vol. 17, No. 7, pp. 366-368.

Spong, M. ; Hutchinson, S. \& Vidyasagar, M. (2006) Robot Modeling and Control. John Wiley \& Sons, Inc. Hoboken, NJ.

Sul, O. ; Falvo, M. ; Taylor, R. ; Washburn, S. \& Superfine, R. (2006). Thermally-actuated untethered impact-driven locomotive microdevices. Applied Physics Letters, Vol. 89, 203512.

Tamaz, S. ; Gourdeau, R. ; Chanu, A. ; Mathieu, J.-B. \& Martel, S. (2008). Real-time MRIbased control of a ferromagnetic core for endovascular navigation. IEEE Transactions on Biomedical Engineering, Vol. 55, No. 7, pp. 1854-1863.

Tea, N. ; Milanovic, V. ; Zincke, C. ; Suehle, J. ; Gaitan, M. ; Zaghloul, M. \& Geist, J. (1997). Hybrid postprocessing etching for CMOS-compatible MEMS. Journal of Microelectromechanical Systems, Vol. 6, No. 4, pp. 363-372.

Tang, W. ; Nguyen, T. ; Judy, M. \& Howe, R. (1990). Electrostatic-comb drive of lateral polysilicon resonators. Sensors and Actuators A, Vol. 21, No. 1-3, pp. 328-331.

Vollmers, K. ; Frutiger, D. ; Kratochvil, B. \& Nelson, B. (2008). Wireless resonant magnetic microactuator for untethered mobile microrobots. Applied Physics Letters, Vol. 92, 144103.

Wang, L.-P. (2003). Design, fabrication and measurement of high-sensitivity piezoelectric microelectromechanical systems accelerometers. Journal of Microelectromechanical Systems, Vol. 12, pp. 433-439.

Wood, R. J. ; Avadhanula, S. ; Menon, M. \& Fearing, R. S. (2003). Microrobotics using composite materials: the micromechanical flying insect thorax. Proceedings of the 2003 IEEE International Conference on Robotics and Automation, pp. 1842-1849, Taipei, Taiwan, Sept. 14-19, 2003.

Yamahata, C. ; Collard, D. ; Domenget, A. ; Hosogi, M. ; Kumemura, M. ; Hashiguchi, G. \& Fujita, H. (2008). Silicon nanotweezers with subnanometer resolution for the micromanipulation of biomolecules. Journal of Microelectromechanical Systems, Vol. 17, No. 3, pp. 623-631.

Yamazaki, A. ; Sendoh, M. ; Ishiyama, K. ; Aria, K. I. ; Kato, R. ; Nakano, M. \& Fukunaga, H. (2004). Wireless micro swimming machine with magnetic thin film. Journal of Magnetism and Magnetic Materials, Vol. 272-276, pp. e1741-e1742.

Yasuda, T ; Shimoyama, I. \& Miura, H. (1994). Microrobot actuated by a vibration energy field. Sensors and Actuators A, Vol. 43, pp. 366-370.

Yasuda, T; Shimoyama, I. \& Miura, H. (1995). Microrobot locomotion in a mechanical vibration field. Advanced Robotics, Vol. 9, pp. 165-176.

Yesin, K. B.; Vollmers, K. \& Nelson, B. J. (2006). Modeling and control of untethered biomicrorobots in a fluidic environment using electromagnetic fields. International Journal of Robot Research, Vol. 25, pp. 527-534. 


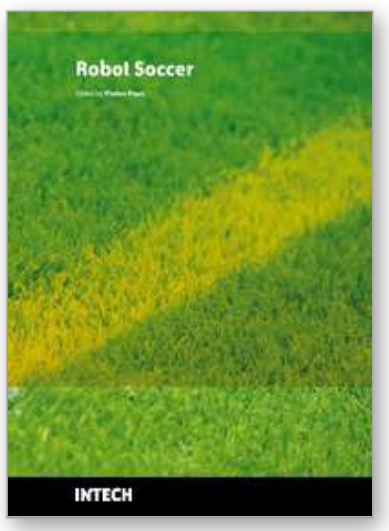

\author{
Robot Soccer \\ Edited by Vladan Papi
}

ISBN 978-953-307-036-0

Hard cover, 348 pages

Publisher InTech

Published online 01, January, 2010

Published in print edition January, 2010

The idea of using soccer game for promoting science and technology of artificial intelligence and robotics was presented in the early 90 s of the last century. Researchers in many different scientific fields all over the world recognized this idea as an inspiring challenge. Robot soccer research is interdisciplinary, complex, demanding but most of all, fun and motivational. Obtained knowledge and results of research can easily be transferred and applied to numerous applications and projects dealing with relating fields such as robotics, electronics, mechanical engineering, artificial intelligence, etc. As a consequence, we are witnesses of rapid advancement in this field with numerous robot soccer competitions and a vast number of teams and team members. The best illustration is numbers from the RoboCup 2009 world championship held in Graz, Austria which gathered around 2300 participants in over 400 teams from 44 nations. Attendance numbers at various robot soccer events show that interest in robot soccer goes beyond the academic and R\&D community. Several experts have been invited to present state of the art in this growing area. It was impossible to cover all aspects of the research in detail but through the chapters of this book, various topics were elaborated. Among them are hardware architecture and controllers, software design, sensor and information fusion, reasoning and control, development of more robust and intelligent robot soccer strategies, Al-based paradigms, robot communication and simulations as well as some other issues such as educational aspect. Some strict partition of chapter in this book hasn't been done because areas of research are overlapping and interweaving. However, it can be said that chapters at the beginning are more system-oriented with wider scope of presented research while later chapters generally deal with some more particular aspects of robot soccer.

\title{
How to reference
}

In order to correctly reference this scholarly work, feel free to copy and paste the following:

S. L. Firebaugh, J. A. Piepmeier and C. D. McGray (2010). Soccer at the Microscale: Small Robots with Big Impact, Robot Soccer, Vladan Papi (Ed.), ISBN: 978-953-307-036-0, InTech, Available from: http://www.intechopen.com/books/robot-soccer/soccer-at-the-microscale-small-robots-with-big-impact

\section{INTECH}

open science | open minds

\section{InTech Europe}

University Campus STeP Ri

Slavka Krautzeka 83/A

51000 Rijeka, Croatia

Phone: +385 (51) 770447

\section{InTech China}

Unit 405, Office Block, Hotel Equatorial Shanghai

No.65, Yan An Road (West), Shanghai, 200040, China

中国上海市延安西路65号上海国际贵都大饭店办公楼 405 单元

Phone: +86-21-62489820 
Fax: +385 (51) 686166

Fax: +86-21-62489821

www.intechopen.com 
(C) 2010 The Author(s). Licensee IntechOpen. This chapter is distributed under the terms of the Creative Commons Attribution-NonCommercial-ShareAlike-3.0 License, which permits use, distribution and reproduction for non-commercial purposes, provided the original is properly cited and derivative works building on this content are distributed under the same license. 